\title{
Study of Multi-Tariff Influence on the Distributed Generation Remuneration
}

\author{
Cátia Silva ${ }^{1}$, Pedro Faria $^{1}$ and Zita Vale ${ }^{1}$ \\ ${ }^{1}$ GECAD - Research Group on Intelligent Engineering and Computing for Advanced Innova- \\ tion and Development, IPP - Polytechnic Institute of Porto, \\ Rua DR. Antonio Bernardino de Almeida, 431, 4200-072 Porto, Portugal; \\ cvcds@isep.ipp.pt; pnfeisep.ipp.pt; zav@isep.ipp.pt
}

\begin{abstract}
The energy market, with the introduction of the smart grids concept, opens the door to small distributed energy resources. However, these resources introduce an added level of difficulty to market management, requiring an entity to aggregate and manage them optimally. This paper proposes an approach that integrates these small resources. The methodology is composed of optimal scheduling, aggregation and remuneration based on aggregation. The method chosen for aggregation is k-means. In relation to previous works, the innovation goes through the multi-period and the comparison that this can have in the formation of groups. Thus, three scenarios were created: Whole Week, Work Days and Weekend. Profiles were added for 548 units of DG. The justification for the formation of groups will be a fairer remuneration and according to the contribution of each resource to the management of the network.
\end{abstract}

Keywords: Aggregation, Clustering, Distributed Generation.

\section{Introduction}

Bidirectional communication will improve the electric distribution network and add great value to the future energy market, [1]. The concept of Smart Grids will introduce new opportunities for small-scale resources, so far without any information about the transactions that existed in the network. Consumers will be able to enter Demand Response (DR) programs where they can respond to real-time signals to change their consumption, [2]. There is also an incentive to use distributed generation (DG) units, not only because of their environmental benefits but also because can help reduce transport losses. Although, this resources will introduce uncertainty to the management of the network being necessary an entity to manage them in a proper way Virtual Power Player (VPP), [3]. The need for tools and models that are able to handle this type and amount of information will be needed. Several works use artificial intel-

The present work was done and funded in the scope of the following projects: COLORS Project and UID/EEA/00760/2019 funded by FEDER Funds through COMPETE program and by National Funds through FCT. 
ligence in this type of models [4]-[5] and the authors propose a management methodology to assist VPP in their task. This paper is the development of previous work, [6]. In the first place, optimal scheduling of resources is proposed, and after an aggregation of resources through a clustering method and final remuneration through a group tariff. In this paper, the aggregation will be done for several time periods and the goal is to understand, testing different numbers of groups, which is the most beneficial situation for VPP. The remuneration made in this way ensures that all resources are remunerated fairly. In this way it is possible to motivate the continued collaboration of resources with VPP.

Section I presents a brief introduction to the theme developed throughout the Paper. The second section details the proposed methodology. Section III presents the case study and section IV the results obtained. Finally, section V presents the conclusions obtained.

\section{Methodology}

As already mentioned, the objective of this methodology is to optimally manage all the small resources associated with the entity that aggregate them. This approach will have several advantages: VPP will have enough energy to enter the market and these resources, which until now had no direct contact with the energy market, could have. Fig. 1 presents a scheme of the methodology proposed by the authors.

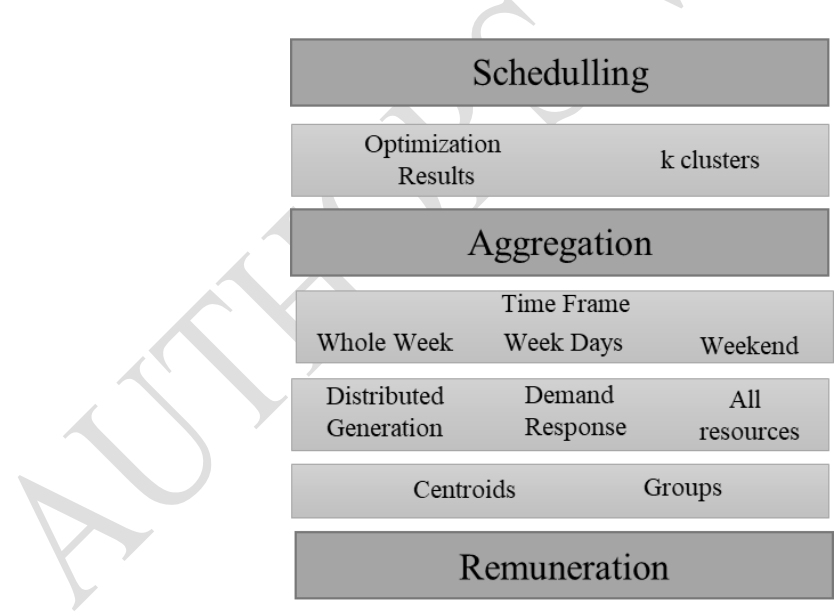

Fig. 1. Proposed Methodology

The first phase, Scheduling, is an optimization problem in which the main objective is to minimize operating costs. The resources that may be associated with the VPP are consumers belonging to DR programs - both incentive based (IDR) and real time pricing (RTP), DG units and suppliers. In the event that DG resources do not meet consumers' needs, suppliers will be used. This optimization is subject to price 
and operating restrictions as well as operational restrictions that are imposed by the VPP to achieve its objectives.

The aggregation phase is in focus in this paper since the objective of this study is to aggregate in three different temporal situations the resources - Whole Week (WW), Work Days (WD) and Weekend (W) and realize which will be the most beneficial. Thus, the input data of this phase are the results of the previous optimization and the number of groups that the VPP intends to see formed - $\mathrm{k}$. The proposal will be the use of a clustering method to accomplish this task. The method selected was k-means. This algorithm defines that at each iteration the distance between the points in the database and the center of each group is calculated, with the said point being assigned to the group with the lowest distance. The aggregation is done separately in this paper, that is, each resource is aggregated by type - DR or DG.

Finally, the remuneration phase is essential in this method. The groups formed served to provide a fair remuneration for the resources and to encourage them to continue to collaborate with the VPP. The authors proposed the remuneration through the maximum tariff for each group. For example, a resource that has a low rate will receive the same way as the rest of the group.

\section{Case Study}

The proposed methodology will be studied through the case study presented in this section. The database belongs to a distribution network of $30 \mathrm{kV}$, with a maximum capacity of 90MVA. The VPP is responsible for specifying different characteristics for each resource, in order to obtain proper results for this methodology. There are five types of consumers: Domestic, Small Commerce, Medium Commerce, Large Commerce and Industrial. In total there are 20310 consumers and a total capacity of $21354.36 \mathrm{kWh}$.

As for DG units, there are seven different ones: Small Hydro, Waste-to-energy, Wind, Photovoltaic, Biomass, Fuell Cell and Co-generation. The number of units is 548 and the total capacity is $25388.79 \mathrm{kWh}$. Consumer profiles, such as DG units and Consumers from DR programs, are for periods of 15 minutes and for one week - January 2-8, 2018. Fig.2. shows how the different scenarios will be studied.

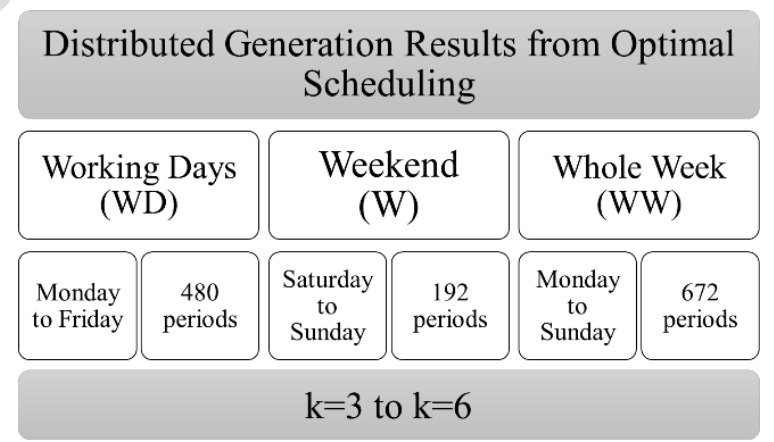

Fig. 2. Formation of case study 


\section{$4 \quad$ Results}

This section presents the results for the second phase of the methodology. This phase was performed using software $\mathrm{R}$ and using the k-means as clustering method. Due to the space limitation, it will not be possible to exhaustively analyze all $\mathrm{k}$ chosen or all resources. Thus, only $\mathrm{k}=6$ will be developed in more detail. The resources chosen for this study were the DG units.

The k-means method used had as output: the group associated with each resource analyzed and the centroid of the group. Fig. 3 presents the centroids for each group found. The centroid value allows us to estimate the mean value of the reduced power by the elements of a given group. This value may be very useful for VPP in the case of adding new resources to existing groups.

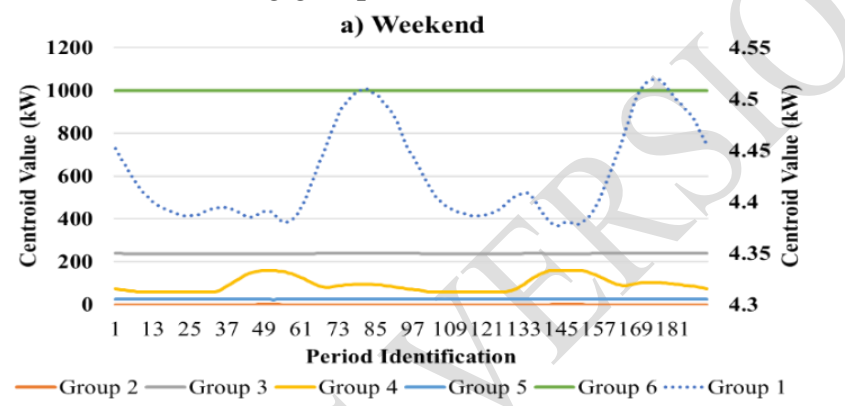

b) Whole Week

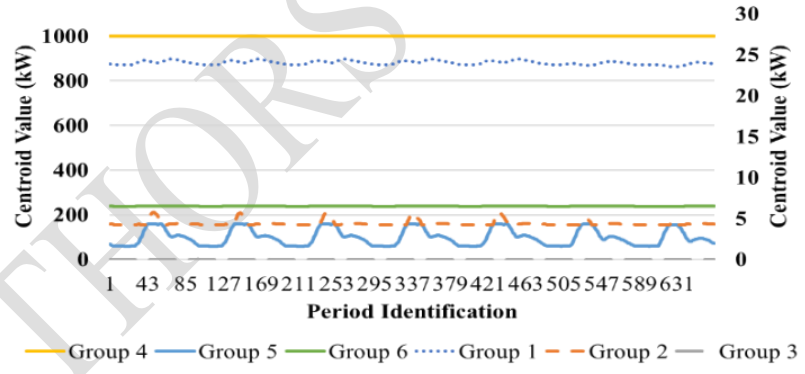

c) Week Days

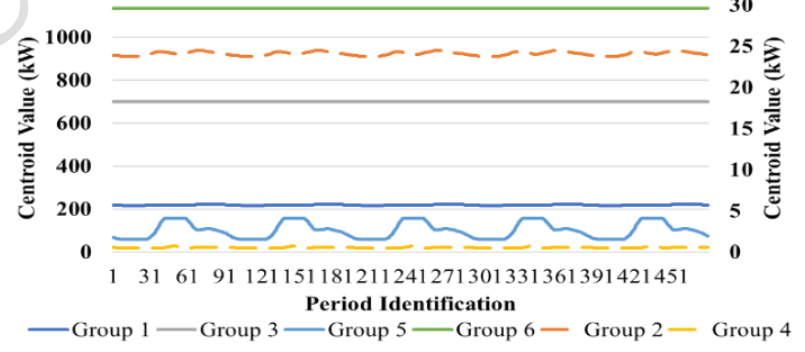

Fig. 3. Centroid value per group

The figures have two y-axis in order to show all curves, due to the difference in scale. Thus, to differentiate the curves from each other, the dashed lines are from the second 
axis. After analyzing the figure, it is possible to conclude that for all scenarios there is the possibility of dividing the groups into three different cases: there are groups with small reductions (below 30kW), medium reductions (between 60 and $250 \mathrm{~kW}$ ) and the largest reductions $(1000 \mathrm{~kW})$.

Table 1 shows the compensation figures for groups and totals. These values were calculated through remuneration with the maximum tariff of a given group by the contribution of each of the resources of DG belonging to that group.

Table 1. Remuneration per group and total

\begin{tabular}{|c|c|c|c|c|}
\hline & Group & WW (m.u.) & WD (m.u.) & W (m.u.) \\
\hline \multirow{4}{*}{$\mathrm{k}=3$} & 1 & 1276923.97 & 915049.45 & 93600.00 \\
\hline & 2 & 218354.66 & 156038.80 & 361874.52 \\
\hline & 3 & 327600.00 & 234000.00 & 62315.86 \\
\hline & Total & 1822878.63 & 1305088.25 & 517790.38 \\
\hline \multirow{5}{*}{$\mathrm{k}=4$} & 1 & 26524.56 & 19089.73 & 62315.86 \\
\hline & 2 & 427620.15 & 234000.00 & 93600.00 \\
\hline & 3 & 327600.00 & 306406.44 & 121213.71 \\
\hline & 4 & 218354.66 & 156038.80 & 7434.84 \\
\hline & Total & 1000099.37 & 715534.97 & 284564.40 \\
\hline \multirow{6}{*}{$\mathrm{k}=5$} & 1 & 26524.56 & 138259.33 & 93600.00 \\
\hline & 2 & 327600.00 & 98280.00 & 35896.10 \\
\hline & 3 & 100346.54 & 19089.73 & 7434.84 \\
\hline & 4 & 125879.33 & 300710.97 & 28670.44 \\
\hline & 5 & 419643.99 & 159120.00 & 118933.02 \\
\hline & Total & 999994.42 & 715460.03 & 284534.40 \\
\hline \multirow{7}{*}{$\mathrm{k}=6$} & 1 & 414532.96 & 132638.80 & 2449.95 \\
\hline & 2 & 8823.16 & 296881.62 & 69.69 \\
\hline & 3 & 0.00 & 98280.00 & 62315.86 \\
\hline & 4 & 327600.00 & 19089.73 & 3465.02 \\
\hline & 5 & 12729.55 & 9264.53 & 117651.34 \\
\hline & 6 & 218354.66 & 159120.00 & 93600.00 \\
\hline & Total & 982040.32 & 715274.68 & 279551.86 \\
\hline
\end{tabular}

Through the analysis of Table 1 , it was concluded that the case $\mathrm{k}=6$ for all scenarios obtains the lowest values of total remuneration. In order to prove the viability of the proposed methodology, different types of remuneration were test and compared. The Table 2 shows the final remuneration for other methods. In this way, method 1 represents the remuneration in a individual way, method 2 uses a formula present by [7] and method 3 the remuneration with the proposed methodology for $k=6$.

Table 2. Remuneration per group and total

\begin{tabular}{c|c|c|c}
\hline \hline Method & WW (m.u.) & WD (m.u.) & W (m.u.) \\
\hline \hline 1 & 978404.56 & 698482.79 & 279921.77 \\
\hline 2 & 685879.51 & 489917.36 & 195962.15 \\
\hline 3 & 982040.32 & 715274.68 & 279551.86 \\
\hline \hline
\end{tabular}


After analyzing the Table 2, the method 2 got the lowest value of remuneration. This formula depends has a parameter that depends of the resource. In this way, depending on fluctuation of this value, the incentive can change. The first and third method have a final remuneration value more similar but, the last one has the higher value. Since the main purpose of this phase is to reward fairly and still motivate the resource to the continuous, the higher the incentive is, the greater the possibility of the participation of the resource. In this way, regardless the disposition of the resource to contribute, it will always receive according to what has been reduced, through the maximum tariff of the group in which it is inserted.

\section{Conclusions}

This paper presents an approach that assists VPP in the task of optimally managing small resources, such as DG units and DR consumers. The focus was on aggregating resources using the clustering algorithm functionalities in the formation of groups in which their elements would have similar characteristics. This would be useful for the remuneration of the resources associated with the VPP, since, by remunerating them by trained groups, they would receive in a fairer way because a specific tariff would be created. Previous work covered this work for only one period. The innovation of this paper is to introduce the concept of multiperiod to perceive the influence of this in the creation of the groups and in the final remuneration of the resources.

\section{References}

[1] N. Yu, T. Wei, and Q. Zhu, "From passive demand response to proactive demand participation," IEEE Int. Conf. Autom. Sci. Eng., vol. 2015-Octob, pp. 1300-1306, 2015.

[2] J. M. Lujano-Rojas, C. Monteiro, R. Dufo-López, and J. L. Bernal-Agustín, "Optimum residential load management strategy for real time pricing (RTP) demand response programs," Energy Policy, vol. 45, pp. 671-679, 2012.

[3] P. Faria, J. Spínola, and Z. Vale, "Aggregation and Remuneration of Electricity Consumers and Producers for the Definition of Demand-Response Programs," IEEE Trans. Ind. Informatics, vol. 12, no. 3, pp. 952-961, 2016.

[4] A. Chaouachi, R. M. Kamel, R. Andoulsi, and K. Nagasaka, "Multiobjective Intelligent Energy Management for a Microgrid," IEEE Trans. Ind. Electron., vol. 60 , no. 4, pp. 1688-1699, 2013.

[5] Z. A. Vale, H. Morais, and H. Khodr, "Intelligent multi-player smart grid management considering distributed energy resources and demand response," in IEEE PES General Meeting, 2010, pp. 1-7.

[6] C. Silva, P. Faria, and Z. Vale, "Clustering Support for an Aggregator in a Smart Grid Context," in 18th International Conference on Hybrid Intelligent Systems (HIS), 2018.

[7] N. G. Cabrera and G. Gutierrez-Alcaraz, "Evaluating demand response programs based on demand management contracts," in IEEE Power and Energy Society General Meeting, 2012, pp. 1-6. 